\title{
Non-symmetric hitting distributions on the hyperbolic half-plane and subordinated perpetuities
}

Paolo Baldi, Enrico Casadio Tarabusi, Alessandro Figà-Talamanca and Marc Yor

Abstract. We study the law of functionals whose prototype is $\int_{0}^{+\infty} e^{B_{s}^{(\nu)}} d W_{s}^{(\mu)}$, where $B^{(\nu)}, W^{(\mu)}$ are independent Brownian motions with drift. These functionals appear naturally in risk theory as well as in the study of invariant diffusions on the hyperbolic half-plane. Emphasis is put on the fact that the results are obtained in two independent, very different fashions (invariant diffusions on the hyperbolic half-plane and Bessel processes).

\section{Introduction.}

Let $W_{t}, B_{t}$ be two independent one-dimensional Brownian motions, and set

$$
W_{t}^{(\mu)}=W_{t}-\mu t, \quad B_{t}^{(\nu)}=B_{t}-\nu t,
$$

where $\nu>0$ and $\mu \in \mathbb{R}$. In this paper we prove some results concerning the distribution of the random variable.

$$
\int_{0}^{+\infty} e^{B_{s}^{(\nu)}} d W_{s}^{(\mu)}
$$


First we prove that it has a density given by

$$
f(x)=c_{\mu, \nu} \frac{e^{-2 \mu \arctan x}}{\left(1+x^{2}\right)^{\nu+1 / 2}},
$$

which belongs to the type IV family of Pearson distributions. The functional (1.1) has been much studied because it appears in risk theory. The density (1.2) was derived in [P, Example 3.1], with a proof for $\nu>1$ only; easy derivations for $\nu>0$ in the particular case $\mu=0$ can be found in [BCF, Remark 4.1] (if, in addition, $\nu$ is a half integer see also [AG, p. 32]). Interestingly, random variables as in (1.1) also appear in connection with invariant diffusions on the hyperbolic halfplane $\Pi=\{z \in \mathbb{C}: \operatorname{Im} z>0\}$.

On $\Pi$ consider the diffusion process associated to the infinitesimal generator

$$
L=\frac{y^{2}}{2} \Delta-\mu y \frac{\partial}{\partial x}-\left(\nu-\frac{1}{2}\right) y \frac{\partial}{\partial y},
$$

where the real coefficients $\mu$ and $\nu-1 / 2$ measure the horizontal, respectively vertical component of the drift (positive for leftward and downward drift, negative for rightward and upward drift). The differential operator $L$ is invariant under the orientation-preserving isometries of $\Pi$ that fix the point at infinity $\infty$, that is, under the real affine transformations $z \longmapsto a z+b$ with $a>0$ and $b \in \mathbb{R}$. The diffusion process associated to $L$ corresponds to the stochastic differential equation

$$
\left\{\begin{array}{l}
d X_{t}=Y_{t} d W_{t}-\mu Y_{t} d t \\
d Y_{t}=Y_{t} d B_{t}-\left(\nu-\frac{1}{2}\right) Y_{t} d t
\end{array}\right.
$$

where, as before, $W_{t}, B_{t}$ are independent one-dimensional Brownian motions. The solution of (1.3) with starting point $i y=(0, y)$ is

$$
\left\{\begin{array}{l}
Y_{t}=y e^{B_{t}^{(\nu)}}, \\
X_{t}=\int_{0}^{t} y e^{B_{s}^{(\nu)}} d W_{s}^{(\mu)} .
\end{array}\right.
$$

Consider the hitting distribution of the diffusion associated to $L$ and starting at $x+i y$ on any horizontal line $H_{a}=\{\operatorname{Im} z=a\}$ with $0 \leq a<$ $y$. For $a=0$ the line $H_{a}$ is the boundary portion $\partial \Pi \backslash\{\infty\}$ (in this case the expression "hitting distribution" is a slight abuse of terminology), 
while for $a>0$ it is a horocycle through $\infty$. Thus the law of the random variable (1.1) is the hitting distribution of the diffusion associated to $L$ in $H_{0}$ and starting at $i$. If $a>0$ the hitting distribution is given by the law of the random variable

$$
\int_{0}^{\tau_{a}} y e^{B_{s}^{(\nu)}} d W_{s}^{(\mu)}
$$

where $\tau_{a}=\inf \left\{t \geq 0: Y_{t}=a\right\}$ is the hitting time on $H_{a}$.

In this paper we prove (1.2) and compute the characteristic function of $f$ in two different fashions.

One is based on a computation of the Poisson kernel of the infinitesimal generator associated to the process. Exploiting the invariance, this kernel can be written in terms of a single function of one real variable that satisfies a second-order linear ordinary differential equation and is determined explicitly. Conjugating by the inverse Fourier transform another second-order linear ordinary differential equation is obtained whose solution is a confluent hypergeometric function and the characteristic function of the hitting distribution. This is done in Section 2 .

The second method uses probabilistic techniques (mostly classical properties of Bessel processes) and is the object of Section 4. It is based on the representation formulae (1.3), (1.4), and uncovers interesting relations between Brownian exponential functionals and previous work of Ph. Biane, J. Pitman, and the fourth-named author on Bessel processes (see [PY1], [PY2], and the references therein).

In Section 3 we discuss an alternate derivation of the ordinary differential equation satisfied by the characteristic function, by means of the Feynman-Kac formula.

In Section 5 we prove that, as the parameters $\mu, \nu$ as well as the coordinates of the starting point of the process take their admissible values (namely $\mu \in \mathbb{R}, \nu>0$ and $\operatorname{Im} z>0$ ), the corresponding hitting distributions belong to the domain of attraction (extended domain of attraction for $\nu=1$ ) of nearly all stable laws with exponent $\alpha=\min \{2,2 \nu\}$, for $0<\alpha \leq 2$.

Finally, Section 6 is devoted to the study of the hitting distribution on $H_{a}$ for $y>a>0$. Using the invariance properties of the diffusion process and the strong Markov property it is possible to derive an expression for the characteristic function of this distribution, and to prove that it still belongs to the domain of attraction of a stable law with exponent $\alpha=2 \nu$. However, in this case we are not able to give an explicit expression for the density. 


\section{The hitting distribution on $H_{0}$ and its characteristic func- tion.}

We perform computations both on the hyperbolic half-plane $\Pi$ and the Poincaré disk $D$. They are isomorphic via the Cayley map $z=$ $i(1-w) /(1+w)$ (where $z \in \Pi$ and $w \in D$ ), which corresponds to $\xi=\tan (\phi / 2)$ on the boundaries, with $\xi \in \mathbb{R} \cup\{\infty\}=\partial \Pi$ and $\left\{e^{i \phi}\right.$ : $-\pi<\phi \leq \pi\}=\partial D$.

The density $P(\xi, z)$ at $\xi$ of the hitting distribution on $\mathbb{R}$ of the process associated to the operator $L$ and starting at $z \in \Pi$ is called the Poisson kernel of $L$ in the domain $\Pi$, and satisfies the following conditions:

1) $L_{x, y} P(\xi, x+i y) \equiv 0$ for all $\xi \in \mathbb{R}$;

2) $P(\xi, z)>0$ for all $\xi \in \mathbb{R}$ and $z \in \Pi$;

3) $\int_{\mathbb{R}} P(\xi, z) d \xi=1$ for all $z \in \Pi$;

4) $\lim _{y \rightarrow 0^{+}} P(\xi, x+i y)=0$ if $\xi \neq x$ and $\xi, x \in \mathbb{R}$.

Since $L$ is invariant under the maps $z \longmapsto a z+b$, then so is the measure $P(\xi, z) d \xi$ on $\mathbb{R}$ for the diagonal action of the same maps, that is, $P(\xi, z)=a P(a \xi+b, a z+b)$. Setting $f(x)=P(x, i)$, we therefore have

$$
P(\xi, x+i y)=\frac{1}{y} P\left(\frac{\xi-x}{y}, i\right)=\frac{1}{y} f\left(\frac{\xi-x}{y}\right) .
$$

In other words, the hitting distribution with arbitrary starting point is obtained, by a simple rescaling, from the one starting at $i$.

The differential operator on $D$ corresponding to $L$ is invariant under the maps $w \longmapsto((1+a+i b) w+(1-a+i b)) /((1-a-i b) w+$ $(1+a-i b))$. Its Poisson kernel $Q$ satisfies

$$
\begin{aligned}
Q(\phi, w)= & \frac{a\left(1+\tan ^{2} \frac{\phi}{2}\right)}{1+\left(a \tan \frac{\phi}{2}+b\right)^{2}} \\
& \cdot Q\left(2 \arctan \left(a \tan \frac{\phi}{2}+b\right), \frac{(1+a+i b) w+(1-a+i b)}{(1-a-i b) w+(1+a-i b)}\right),
\end{aligned}
$$

so that, if $g(\phi)=Q(\phi, 0)$, then

$$
Q(\phi, w)=\frac{1-|w|^{2}}{\left|e^{i \phi}-w\right|^{2}} g\left(2 \arctan \frac{|1+w|^{2} \tan \frac{\phi}{2}-2 \operatorname{Im} w}{1-|w|^{2}}\right) .
$$


Furthermore $f(x)=2 g(2 \arctan x) /\left(1+x^{2}\right)$.

Condition 1) can be translated for $f$ using (2.1), then setting $\xi=0$ (since $L$ is autonomous in $x$ this may also be done beforehand) and $y=$ 1. The result is the second-order linear ordinary differential equation $M f=0$, with

$$
\begin{aligned}
M f(x) & =\frac{1+x^{2}}{2} f^{\prime \prime}(x)+\left(\mu+\left(\nu+\frac{3}{2}\right) x\right) f^{\prime}(x)+\left(\nu+\frac{1}{2}\right) f(x) \\
& =\frac{d}{d x}\left(\frac{d}{d x}\left(\frac{1+x^{2}}{2} f(x)\right)+\left(\mu+\left(\nu-\frac{1}{2}\right) x\right) f(x)\right)
\end{aligned}
$$

proportional to

$$
\frac{d}{d \phi}\left(\left(g^{\prime}(\phi)+\left(\mu+\left(\nu-\frac{1}{2}\right) \tan \frac{\phi}{2}\right) g(\phi)\right) \cos ^{2} \frac{\phi}{2}\right)
$$

if $\phi=2 \arctan x$. The first-order linear equation obtained by equating the expression in square brackets to a constant multiple of $\cos ^{-2}(\phi / 2)$ is solved by

$g(\phi)=\left(\frac{c}{2}+k \int_{0}^{\phi} e^{\mu \phi} \cos ^{-2 \nu-1} \frac{\phi}{2} d \phi\right) e^{-\mu \phi} \cos ^{2 \nu-1} \frac{\phi}{2}, \quad$ with $c, k \in \mathbb{R}$.

Since $P$, whence $f, Q, g$, must be positive by condition 2 ) and since for $\phi \in(-\pi, \pi)$ the above integral takes arbitrarily large values of either sign because $\nu>0$, then $k=0$ and $f$ is given by (1.2).

Since $\int_{-\pi}^{\pi} g=1$ as a consequence of condition 3 ), then by [GR, 3.892.2 and 8.384.1] and the basic properties of the Euler Gamma function we have

$$
\begin{aligned}
c & =c_{\mu, \nu} \\
& =2\left(\int_{-\pi}^{\pi} e^{-\mu \phi} \cos ^{2 \nu-1} \frac{\phi}{2} d \phi\right)^{-1} \\
& =\frac{2^{2 \nu-1}\left|\Gamma\left(\frac{1}{2}+\nu-i \mu\right)\right|^{2}}{\pi \Gamma(2 \nu)}
\end{aligned}
$$

In particular, by [GR, 8.332.2-3]

$$
c_{\mu, 1 / 2}=\frac{\mu}{\sinh \mu \pi}, \quad c_{\mu, 1}=\frac{\frac{1}{2}+2 \mu^{2}}{\cosh \mu \pi},
$$


and, more generally,

$$
c_{\mu, \nu}= \begin{cases}\frac{2^{2 \nu-1}}{(2 \nu-1) ! \mu \sinh \mu \pi} \prod_{j=0}^{\nu-1 / 2}\left(j^{2}+\mu^{2}\right), & \text { if } \nu=\frac{1}{2}, \frac{3}{2}, \frac{5}{2}, \ldots, \\ \frac{2^{2 \nu-1}}{(2 \nu-1) ! \cosh \mu \pi} \prod_{j=0}^{\nu-1}\left(\left(j+\frac{1}{2}\right)^{2}+\mu^{2}\right), & \text { if } \nu=1,2,3, \ldots,\end{cases}
$$

as can also be checked by elementary means from the integral expression of $c_{\mu, \nu}$. On the other hand, for $\mu=0$ from [GR, 8.335.1] we have

$$
c_{0, \nu}=\frac{\Gamma\left(\frac{1}{2}+\nu\right)}{\sqrt{\pi} \Gamma(\nu)} .
$$

We now compute the characteristic function of the hitting distribution. Again by invariance, the expression for an arbitrary starting point $x+$ $i y \in \Pi$ can be derived from the special case of starting point $i$. Indeed, if $u=\mathcal{F}^{-1} f$ is the inverse Fourier transform of $f$, then by (2.1) the required characteristic function is $\mathcal{F}^{-1} P(\cdot, x+i y)=e^{i \lambda x} u(\lambda y)$.

We have $u(-\lambda)=\overline{u(\lambda)}$ because $f$ is real-valued, and $u(0)=1$ by condition 3). Moreover, for $k=0,1,2$ the function $x^{k} f^{(k)}(x)$ is integrable, whence $\lambda^{k} u^{(k)}(\lambda)$ (exists and) is continuous, and vanishes at infinity; in particular, $u$ is continuous on $\mathbb{R}$ and twice continuously differentiable outside 0 , and vanishes at infinity. Thus $u$ is in the kernel of the operator $N=\mathcal{F}^{-1} M \mathcal{F}$, given by

$$
N u(\lambda)=\frac{\lambda^{2}}{2} u^{\prime \prime}(\lambda)-\left(\nu-\frac{1}{2}\right) \lambda u^{\prime}(\lambda)-\left(\frac{\lambda^{2}}{2}+i \mu \lambda\right) u(\lambda) .
$$

With the change of variables $v(w)=e^{w / 2} u(w / 2)$ the equation $N u=0$ becomes

$$
\begin{aligned}
& w v^{\prime \prime}(w)+(b-w) v^{\prime}(w)-a v(w)=0, \\
& \text { where }\left\{\begin{array}{l}
a=\frac{1}{2}-\nu+i \mu, \\
b=1-2 \nu
\end{array}\right.
\end{aligned}
$$

This is a confluent hypergeometric equation in one of its standard forms [EMOT, Chapter VI], [T], and its solutions are called confluent hypergeometric functions. One solution for $w>0$ is the Tricomi $\Psi$-function, defined as in [EMOT, 6.11.(13)] by

$$
\Psi(a, b ; w)=\frac{2^{1-b} \Gamma(1-a) e^{w / 2}}{\pi} \int_{0}^{\pi / 2} \cos \left(\frac{w}{2} \tan \theta+(2 a-b) \theta\right) \cos ^{-b} \theta d \theta .
$$


For this formula to hold it is required that $\operatorname{Re} b<1$ and $a$ is not a positive integer, both of which hold for $a, b$ given in (2.5). Since $\Psi(a, b ; w)$ has a finite non-zero limit for $w \rightarrow 0^{+}$and since

$$
\lim _{w \rightarrow+\infty} e^{-w / 2} \Psi(a, b ; w)=0
$$

[EMOT, 6.13.(1)], then the solution of $N u=0$ we are looking for is, for $\lambda>0$, a multiple of $e^{-\lambda} \Psi(a, b ; 2 \lambda)$. After some obvious manipulations (2.3) gives

$$
\frac{1}{2 c_{\mu, \nu}}=\int_{0}^{\pi / 2} \cos (2 i \mu \theta) \cos ^{2 \nu-1} \theta d \theta,
$$

so that

$$
\begin{aligned}
\Psi\left(\frac{1}{2}-\nu+i \mu, 1\right. & -2 \nu ; 0) \\
& =\frac{2^{2 \nu} \Gamma\left(\frac{1}{2}+\nu-i \mu\right)}{\pi} \int_{0}^{\pi / 2} \cos (2 i \mu \theta) \cos ^{2 \nu-1} \theta d \theta \\
& =\frac{1}{2 c_{\mu, \nu} \pi} 2^{2 \nu} \Gamma\left(\frac{1}{2}+\nu-i \mu\right) \\
& =\frac{\Gamma(2 \nu)}{\Gamma\left(\frac{1}{2}+\nu+i \mu\right)} .
\end{aligned}
$$

We summarize the results of this section:

Proposition 2.1. For every $\nu>0$ and $\mu \in \mathbb{R}$, as $t \longrightarrow+\infty$ the distribution of $X_{t}$ with starting point $i$ converges to the probability defined by the density (1.2). Its characteristic function is

$$
\frac{\Gamma\left(\frac{1}{2}+\nu+i \mu\right)}{\Gamma(2 \nu)} e^{-\lambda} \Psi\left(\frac{1}{2}-\nu+i \mu, 1-2 \nu ; 2 \lambda\right),
$$

where $\Psi$ is the Tricomi $\Psi$-function. 


\section{Use of the Feynman-Kac formula.}

We now prove in a different way that the characteristic function $u$ of $X_{\infty}$ with starting point $\left(X_{0}, Y_{0}\right)=(0,1)$, i.e., thanks to $(1.1)$, the function

$$
u(\lambda)=\mathbf{E}_{0,1}\left[e^{i \lambda X_{\infty}}\right]=\mathbf{E}\left[\exp \left(i \lambda \int_{0}^{\infty} e^{B_{s}^{(\nu)}} d W_{s}^{(\mu)}\right)\right],
$$

is in the kernel of the ordinary differential operator (2.4). Henceforth we denote by $P_{x, y}$ the law of the diffusion with starting point $\left(X_{0}, Y_{0}\right)=(x, y)$ and by $\mathbf{E}_{x, y}$ the corresponding expectation. Since the $Y$-component is independent from $W$, then

$$
\begin{aligned}
u(\lambda) & =\mathbf{E}_{0,1}\left[\exp \left(i \lambda \int_{0}^{\infty} Y_{s} d W_{s}-i \mu \lambda \int_{0}^{\infty} Y_{s} d s\right)\right] \\
& =\mathbf{E}_{0,1}\left[\exp \left(-\frac{\lambda^{2}}{2} \int_{0}^{\infty} Y_{s}^{2} d s-i \mu \lambda \int_{0}^{\infty} Y_{s} d s\right)\right] \\
& =\mathbf{E}\left[\exp \int_{0}^{\infty}-\left(\frac{\left(\lambda e^{B_{s}^{(\nu)}}\right)^{2}}{2}+i \mu \lambda e^{B_{s}^{(\nu)}}\right) d s\right] \\
& =\mathbf{E}_{0, \lambda}\left[\exp \int_{0}^{\infty} G\left(Y_{s}\right) d s\right]
\end{aligned}
$$

where $G(y)=-y^{2} / 2-i \mu y$.

Proposition 3.1. Let $N$ be given by (2.4), and let $u(\lambda)$ be a solution for $\lambda>0$ of $N u=0$ such that

$$
\begin{aligned}
& \lim _{\lambda \rightarrow 0^{+}} u(\lambda)=1, \\
& \lim _{\lambda \rightarrow \infty} u(\lambda)=0 .
\end{aligned}
$$

Extend $u$ to the negative half-line by setting $u(\lambda)=\overline{u(-\lambda)}$ for $\lambda<0$. Then $u(\lambda)=\mathbf{E}_{0,1}\left[e^{i \lambda X_{\infty}}\right]$.

(Unlike in the previous section, we require $\lim _{\lambda \rightarrow \infty} \lambda^{k} u(\lambda)=0$ only for $k=0$.) 
Proof. For $0<a<1$ let

$$
\sigma_{a}=\inf \left\{t \geq 0: Y_{t}=a \text { or } Y_{t}=\frac{1}{a}\right\}
$$

be the exit time of $Y_{t}$ from the interval $(a, 1 / a)$. Then the Feynman-Kac formula gives

$$
u(\lambda)=\mathbf{E}_{0, \lambda}\left[u\left(Y_{\sigma_{a}}\right) \exp \int_{0}^{\sigma_{a}} G\left(Y_{s}\right) d s\right]
$$

As $a \longrightarrow 0$ we have $\sigma_{a} \longrightarrow+\infty$ and $u\left(Y_{\sigma_{a}}\right) \longrightarrow u(0)=1$ almost surely.

\section{A probabilistic computation of the hitting distribution.}

We shall now compute again the law of $\int_{0}^{\infty} e^{B_{s}^{(\nu)}} d W_{s}^{(\mu)}$, as a consequence of the following three simple observations.

1) For a fixed real number $x$ (the starting point), consider the two processes

$$
\begin{aligned}
& X_{t}^{(\mu, \nu)}=e^{B_{t}^{(\nu)}} x+\int_{0}^{t} e^{B_{s}^{(\nu)}} d W_{s}^{(\mu)}, \\
& \widetilde{X}_{t}^{(\mu, \nu)}=e^{B_{t}^{(\nu)}}\left(x+\int_{0}^{t} e^{-B_{s}^{(\nu)}} d W_{s}^{(\mu)}\right) .
\end{aligned}
$$

Then $X_{t}^{(\mu, \nu)}, \widetilde{X}_{t}^{(\mu, \nu)}$ have the same law for every fixed $t$ (although the two processes do not have the same law). More generally this holds whenever $B, W$ are independent Lévy processes [CPY, Lemma 2.3].

2 ) The process $\left(\widetilde{X}_{t}^{(\mu, \nu)}, t \geq 0\right)$ is a diffusion process with generator

$$
M^{*}=\frac{1+x^{2}}{2} \frac{d^{2}}{d x^{2}}-\left(\mu+\left(\nu-\frac{1}{2}\right) x\right) \frac{d}{d x},
$$

the adjoint of the operator $M$ given in (2.2).

3) The distribution at time $t$ of this diffusion process converges to the invariant distribution, whose density $f(x)$ is given in (1.2). 
Proof of 2). By Itô's formula

$$
\begin{aligned}
d \widetilde{X}_{t}^{(\mu, \nu)} & =\widetilde{X}_{t}^{(\mu, \nu)}\left(d B_{t}^{(\nu)}+\frac{d t}{2}\right)+e^{B_{t}^{(\nu)}} e^{-B_{t}^{(\nu)}} d W_{t}^{(\mu)} \\
& =\widetilde{X}_{t}^{(\mu, \nu)} d B_{t}+d W_{t}-\left(\mu+\left(\nu-\frac{1}{2}\right) \widetilde{X}_{t}^{(\mu, \nu)}\right) d t
\end{aligned}
$$

from which one derives easily that $\widetilde{X}^{(\mu, \nu)}$ is a diffusion process with a generator as stated.

Proof of 3$)$. From 1), $\widetilde{X}_{t}^{(\mu, \nu)}$ converges in law as $t \longrightarrow+\infty$, since $\widetilde{X}_{t}^{(\mu, \nu)} \stackrel{\text { law }}{\simeq} X_{t}^{(\mu, \nu)}$, and $X_{t}^{(\mu, \nu)} \longrightarrow X_{\infty}^{(\mu, \nu)}$. It is easy to see that the limit distribution is invariant, that is, it is annihilated by $M$, whence it necessarily coincides with $f$.

As remarked in Section 3, the hitting distribution on $H_{0}$ under $P_{0, y}$ is the law of the random variable $\int_{0}^{\infty} y e^{B_{s}^{(\nu)}} d W_{s}^{(\mu)}$. If we set

$$
A_{\infty}^{(\nu)}=\int_{0}^{\infty} e^{2 B_{s}^{(\nu)}} d s, \quad A_{\infty}^{(\nu, 1)}=\int_{0}^{\infty} e^{B_{s}^{(\nu)}} d s
$$

then $X_{\infty}$ can be written in the form of a subordinated perpetuity as

$$
X_{\infty}=\gamma_{A_{\infty}^{(\nu)}}-\mu A_{\infty}^{(\nu, 1)}
$$

where $\gamma$ is a Brownian motion independent of $B, W$. It is thus clear that the law of $X_{\infty}$ is the same as that of

$$
Z \sqrt{A_{\infty}^{(\nu)}}-\mu A_{\infty}^{(\nu, 1)}
$$

where $Z$ is an $N(0,1)$ random variable, independent of $B, W$. If $h$ is any bounded Borel function on $\mathbb{R}$, then

$$
\begin{aligned}
\mathbf{E}\left[h\left(X_{\infty}\right)\right] & =\mathbf{E}\left[h\left(Z \sqrt{A_{\infty}^{(\nu)}}-\mu A_{\infty}^{(\nu, 1)}\right)\right] \\
& =\int_{\mathbb{R}} \frac{e^{-z^{2} / 2}}{\sqrt{2 \pi}} \mathbf{E}\left[h\left(z \sqrt{A_{\infty}^{(\nu)}}-\mu A_{\infty}^{(\nu, 1)}\right)\right] d z \\
& =\mathbf{E}\left[\int_{\mathbb{R}} \frac{h(x)}{\sqrt{2 \pi A_{\infty}^{(\nu)}}} e^{-\left(x+\mu A_{\infty}^{(\nu, 1)}\right)^{2} /\left(2 A_{\infty}^{(\nu)}\right)} d x\right] .
\end{aligned}
$$


Since $h$ is arbitrary, this yields a representation formula for the density $f$ of $X_{\infty}$ :

Theorem 4.1. We have

$$
f(x)=\mathbf{E}\left[\frac{1}{\sqrt{2 \pi A_{\infty}^{(\nu)}}} e^{-\left(x+\mu A_{\infty}^{(\nu, 1)}\right)^{2} /\left(2 A_{\infty}^{(\nu)}\right)}\right] .
$$

Next comes a representation formula of the density $f$ in terms of Bessel processes. The main tool is Lamperti's representation formula for the geometric Brownian motion [RY, Exercise 11.1.28], which states that

$$
e^{B_{s}^{(\nu)}}=R_{A_{s}^{(\nu)}}^{(-\nu)}
$$

where $R^{(-\nu)}$ is a Bessel process with index $-\nu$. Taking $s \longrightarrow+\infty$ in this relation, since the left-hand side tends to 0 one has $R_{A_{\infty}^{(\nu)}}^{(-\nu)}=0$, so that $A_{\infty}^{(\nu)}$ coincides with the first passage time $T_{0}\left(R^{(-\nu)}\right)$ of $R^{(-\nu)}$ by 0 . Moreover one can write

$$
A_{\infty}^{(\nu, 1)}=\int_{0}^{\infty} e^{B_{s}^{(\nu)}} d s=\int_{0}^{\infty} \frac{d A_{s}^{(\nu)}}{e^{B_{s}^{(\nu)}}}=\int_{0}^{\infty} \frac{d A_{s}^{(\nu)}}{R_{A_{s}^{(\nu)}}^{(-\nu)}}=\int_{0}^{T_{0}\left(R^{(-\nu)}\right)} \frac{d u}{R_{u}^{(-\nu)}} .
$$

This can be summarized by stating that

$$
\left(A_{\infty}^{(\nu)}, A_{\infty}^{(\nu, 1)}\right) \stackrel{\text { law }}{\simeq}\left(T_{0}\left(R^{(-\nu)}\right), \int_{0}^{T_{0}\left(R^{(-\nu)}\right)} \frac{d u}{R_{u}^{(-\nu)}}\right) .
$$

Theorem 4.2. Denote by $P^{(\nu)}$ the law of the transient Bessel process $\left(R_{u}^{(\nu)}, u \geq 0\right)$ with dimension $d=2(\nu+1)$ starting at 0 , and define

$$
H_{s}=\int_{0}^{s} \frac{d u}{R_{u}^{(\nu)}} .
$$

Then

$$
f(x)=\sqrt{\frac{2}{\pi}} \mathbf{E}^{(\nu)}\left[\frac{\nu}{R_{1}^{(\nu)}} e^{-\left(x R_{1}^{(\nu)}+\mu H_{1}\right)^{2} / 2}\right] .
$$

In the particular case $\nu=1 / 2$ we have that $\left(R_{u}^{(\nu)}, u \geq 0\right)$ is the 3 -dimensional Bessel process starting at 0 . 
Proof. Recall D. Williams' time reversal result, which states that

$$
\left(R_{T_{0}\left(R^{(-\nu)}\right)-u}^{(-\nu)}, u \leq T_{0}\left(R^{(-\nu)}\right)\right) \stackrel{\text { law }}{\simeq}\left(R_{u}^{(\nu)}, u \leq L_{1}\left(R^{(\nu)}\right)\right),
$$

where the right-hand side denotes the Bessel process with index $\nu$ starting from 0 , and $L_{1}$ its last passage time by 1 . Thus (4.2) may be written

$$
\left(A_{\infty}^{(\nu)}, A_{\infty}^{(\nu, 1)}\right) \stackrel{\text { law }}{\simeq}\left(L_{1}\left(R^{(\nu)}\right), \int_{0}^{L_{1}\left(R^{(\nu)}\right)} \frac{d u}{R_{u}^{(\nu)}}\right) .
$$

It is now sufficient to use a result of absolute continuity between the laws of

$$
\left(\frac{R_{u L_{1}}^{(\nu)}}{\sqrt{L_{1}}}, u \leq 1\right)
$$

and of $\left(R_{u}^{(\nu)}, u \leq 1\right)$, a transient Bessel process starting at 0 [BLY, Théorème 3], [Y, sections 2 and 4$]$.

Techniques based on Bessel processes give also an alternative proof of expression (2.6) for the characteristic function of the hitting distribution.

We assume $y=1$. Lamperti's representation formula (4.1) implies also that $A_{\tau_{x}}^{(\nu)}=T_{x}\left(R^{(-\nu)}\right)$, where we denote by $\tau_{x}$ the hitting time in $x$ of $Y_{t}=e^{B_{t}^{(\nu)}}$ and $T_{x}\left(R^{(-\nu)}\right)$ the hitting time in $x$ of the Bessel process $R^{(-\nu)}$. The same arguments leading to (4.2) give

$$
\left(A_{\tau_{x}}^{(\nu)}, A_{\tau_{x}}^{(\nu, 1)}\right) \stackrel{\text { law }}{\simeq}\left(T_{x}\left(R^{(-\nu)}\right), \int_{0}^{T_{x}\left(R^{(-\nu)}\right)} \frac{d u}{R_{u}^{(-\nu)}}\right) .
$$

Thus for $\theta \in \mathbb{R}$, using [PY1, Proposition 12.2, p. 363] (see [PY2] for more information) and the expression of the density of the law of a Bessel process with index $-\nu$ with respect to the law of a Bessel process with index 0 (see, e.g., [RY, Exercise 11.1.18]), we have

$$
\begin{aligned}
\mathbf{E}\left[e^{i \theta X_{\tau_{x}}}\right] & =\mathbf{E}\left[\exp \left(i \theta \int_{0}^{\tau_{x}} e^{B_{s}^{(\nu)}} d \gamma_{s}-i \theta \mu \int_{0}^{\tau_{x}} e^{B_{s}^{(\nu)}} d s\right)\right] \\
& =\mathbf{E}\left[\exp \left(-\frac{\theta^{2}}{2} \int_{0}^{\tau_{x}} e^{2 B_{s}^{(\nu)}} d s-i \theta \mu \int_{0}^{\tau_{x}} e^{B_{s}^{(\nu)}} d s\right)\right] \\
& =\mathbf{E}_{1}^{(-\nu)}\left[\exp \left(-\frac{\theta^{2}}{2} T_{x}\left(R^{(-\nu)}\right)-i \theta \mu \int_{0}^{T_{x}\left(R^{(-\nu)}\right)} \frac{d u}{R_{u}^{(-\nu)}}\right)\right]
\end{aligned}
$$




$$
=x^{-\nu+1 / 2} \frac{W_{-i \mu, \nu}(2 \theta)}{W_{-i \mu, \nu}(2 \theta x)}
$$

where $W .$, . denotes the Whittaker functions. This gives the characteristic function of the hitting distribution on $H_{x}$. Recalling the relation between the Whittaker and Tricomi $\Psi$-functions [T, 2.4.(5)]

$$
W_{k, \nu}(z)=z^{\nu+1 / 2} e^{-z / 2} \Psi\left(\frac{1}{2}+\nu-k, 1+2 \mu ; z\right)
$$

and a functional property of the $\Psi$ function $[\mathrm{T}, 2.3 .(9)]$

$$
\Psi(a-c+1,2-c ; z)=z^{c-1} \Psi(a, c ; z),
$$

we get

$$
\begin{aligned}
W_{-i \mu, \nu}(2 \theta) & =(2 \theta)^{\nu+1 / 2} e^{-\theta} \Psi\left(\frac{1}{2}+\nu+i \mu, 1+2 \nu ; 2 \theta\right) \\
& =(2 \theta)^{-\nu+1 / 2} e^{-\theta} \Psi\left(\frac{1}{2}-\nu+i \mu, 1-2 \nu ; 2 \theta\right),
\end{aligned}
$$

so that

$$
\begin{aligned}
\mathbf{E}\left[e^{i \theta X_{\tau_{x}}}\right] & =x^{-\nu+1 / 2} \frac{(2 \theta)^{-\nu+1 / 2} e^{-\theta} \Psi\left(\frac{1}{2}-\nu+i \mu, 1-2 \nu ; 2 \theta\right)}{(2 \theta x)^{-\nu+1 / 2} e^{-\theta x} \Psi\left(\frac{1}{2}-\nu+i \mu, 1-2 \nu ; 2 \theta x\right)} \\
& =\frac{e^{-\theta} \Psi\left(\frac{1}{2}-\nu+i \mu, 1-2 \nu ; 2 \theta\right)}{e^{-\theta x} \Psi\left(\frac{1}{2}-\nu+i \mu, 1-2 \nu ; 2 \theta x\right)}
\end{aligned}
$$

This gives the characteristic function of the hitting distribution on the horocycle $H_{x}$. Taking $x \longrightarrow 0^{+}$one gets easily

$$
\mathbf{E}\left[e^{i \theta X_{\infty}}\right]=\frac{e^{-\theta} \Psi\left(\frac{1}{2}-\nu+i \mu, 1-2 \nu ; 2 \theta\right)}{\Psi\left(\frac{1}{2}-\nu+i \mu, 1-2 \nu ; 0\right)}
$$

which is consistent with Proposition 2.1. 


\section{Hitting distributions and stable laws.}

The definitions and the theorem below are taken from $[\mathrm{H}$, sections 5.18 and 5.25].

Definition 5.1. A probability distribution is stable if and only if its characteristic function $\phi$ is of the following form $S(z, c, \alpha, \gamma)$

$$
\phi(t)=\left\{\begin{array}{c}
\exp \left(i z t+c|t|^{\alpha}\left(1+i \gamma \operatorname{sgn}(t) \tan \frac{\alpha \pi}{2}\right)\right), \\
\quad \text { if } 0<\alpha \leq 2 \text { and } \alpha \neq 1, \\
\exp \left(i z t+c|t|^{\alpha}\left(1+i \gamma \operatorname{sgn}(t) \frac{2}{\pi} \log |t|\right)\right), \\
\quad \text { if } \alpha=1,
\end{array}\right.
$$

where $c>0,1 \leq \gamma \leq 1$, and $z \in \mathbb{R}$.

Definition 5.2. A probability law $m_{0}$ is said to belong to the domain of attraction of a stable law $m$ if there exist two sequences of real numbers $\left\{a_{n}\right\}_{n},\left\{b_{n}\right\}_{n}$ such that

$$
\frac{X_{1}+\cdots+X_{n}-b_{n}}{a_{n}} \stackrel{\text { law }}{\longrightarrow} m, \quad \text { as } n \longrightarrow \infty
$$

where $\left\{X_{n}\right\}_{n}$ is a sequence of independent and identically distributed random variables with common law equal to $m_{0}$.

Define

$$
C(\alpha)= \begin{cases}-\Gamma(-\alpha) \cos \frac{\alpha \pi}{2}, & \text { if } 0<\alpha<2 \text { and } \alpha \neq 1 \\ \frac{\pi}{2}, & \text { if } \alpha=1 .\end{cases}
$$

Note that $C(\alpha)>0$ whenever $0<\alpha<2$.

Theorem 5.3. Let $\left\{X_{n}\right\}_{n}$ be a sequence of independent and identically distributed random variables and assume that

$$
\lim _{x \rightarrow \infty} x^{\alpha} P\left(X_{1}>x\right)=a, \quad \lim _{x \rightarrow \infty} x^{\alpha} P\left(X_{1}<-x\right)=b,
$$


where $0<\alpha<2$, and $a, b \geq 0$ with $a+b>0$. Set

$$
\begin{gathered}
m_{n}= \begin{cases}0, & \text { if } 0<\alpha<1, \\
n \mathbf{E}\left[\sin \left(\frac{1}{n} X_{1}\right)\right], & \text { if } \alpha=1, \\
\mathbf{E}\left[X_{1}\right], & \text { if } 1<\alpha<2,\end{cases} \\
c=\alpha(a+b) C(\alpha), \\
\gamma=\frac{b-a}{b+a} .
\end{gathered}
$$

Then

$$
\frac{X_{1}+\cdots+X_{n}-n m_{n}}{n^{1 / \alpha}} \stackrel{\text { law }}{\longrightarrow} m, \quad \text { as } n \longrightarrow \infty,
$$

where $m$ is $S(0, c, \alpha, \gamma)$.

We have determined in the previous sections that the density $f$ of the random variable $X_{\infty}$ is given by (1.2) if the starting point is $i=(0,1)$. If the starting point is $(0, y)$, then the hitting distribution is the same as the law of $y Z$ where $Z$ is distributed according to $f$. We now check that such a distribution belongs to the domain of attraction of a stable law, of which we determine the parameters.

Assume first that $0<\nu<1$. We have

$$
\lim _{x \rightarrow \infty} x^{2 \nu} P(Z>x)=\frac{c_{\mu, \nu}}{2 \nu} e^{-\mu \pi}, \quad \lim _{x \rightarrow \infty} x^{2 \nu} P(Z<-x)=\frac{c_{\mu, \nu}}{2 \nu} e^{\mu \pi},
$$

so that, if the starting point is $i y=(0, y)$, then

$$
\begin{aligned}
& a=\lim _{x \rightarrow \infty} x^{2 \nu} P_{0, y}\left(X_{\infty}>x\right)=\frac{c_{\mu, \nu} y^{2 \nu}}{2 \nu} e^{-\mu \pi}, \\
& b=\lim _{x \rightarrow \infty} x^{2 \nu} P_{0, y}\left(X_{\infty}<-x\right)=\frac{c_{\mu, \nu} y^{2 \nu}}{2 \nu} e^{\mu \pi} .
\end{aligned}
$$

Thus the assumption of Theorem 5.3 is satisfied and this density belongs to the domain of attraction of the stable law $S(0, c, 2 \nu, \gamma)$.

Let us investigate the possible values of the parameters $c, \gamma$. Clearly

$$
c=2 c_{\mu, \nu} y^{2 \nu} C(2 \nu) \cosh \mu \pi, \quad \gamma=\tanh (-\mu \pi) .
$$

Thus the parameter $\gamma$ can take all the values in the range $(-1,1)$, that is, all possible values except the extremal ones \pm 1 . Finally, by tuning the value of $y$, one can make $c$ take any positive value. 
It is clear that if $\nu>1$ then the hitting distribution, having a finite second order moment, belongs to the domain of attraction of a Gaussian law. If $\nu=1$ then a finite second moment does not exist, but it is known [GK, Theorem 35.1] that a probability law $\mu$ belongs to the extended domain of attraction of a Gaussian distribution if and only if

$$
\lim _{x \rightarrow+\infty} \frac{x^{2}\left(1-\int_{-x}^{x} \mu(d y)\right)}{\int_{-x}^{x} y^{2} \mu(d y)}=0 .
$$

It is immediate to check that the above condition is satisfied for the density (1.2) with $\nu=1$. This means that there exist two sequences $\left\{a_{n}\right\}_{n},\left\{b_{n}\right\}_{n}$ of real numbers, with $a_{n}>0$, such that if $\left\{X_{n}\right\}_{n}$ is a sequence of independent, identically distributed random variables with density (1.2) for $\nu=1$, then

$$
\frac{X_{1}+\cdots+X_{n}}{a_{n}}-b_{n} \stackrel{\text { law }}{\longrightarrow} N(0,1)
$$

(although $a_{n}$ is not necessarily equal to $n^{1 / \alpha}$ ).

\section{The case $a>0$.}

Recall that we denote by $\tau_{a}$ the first hitting time of the diffusion associated to $L$ on the horocycle $H_{a}$, with $a>0$. We now show how the characteristic function of the hitting distribution on $H_{a}$ can be derived from that of $X_{\infty}$. This will allow us to prove that the hitting distribution on $H_{a}$ is still in the domain of attraction of a stable law with exponent $2 \nu$, but we are not able to give its density. Denote by $K$ the Fourier transform of the hitting distribution on $H_{0}$ with starting point $i$, that is, with the notation of Section 5 , the distribution of $X_{\infty}$ under $P_{0,1}$. Then the characteristic function with starting point $i y$ is $t \longrightarrow K(y t)$. By conditioning with respect to the $\sigma$-algebra $\mathcal{F}_{\tau_{a}}$ and using the strong Markov property, for $a<y$ one has

$$
\begin{aligned}
K(y t) & =\mathbf{E}\left[\exp \left(i t \int_{0}^{+\infty} y e^{B_{s}^{(\nu)}} d W_{s}^{(\mu)}\right)\right] \\
& =\mathbf{E}_{0, y}\left[\exp \left(i t \int_{0}^{+\infty} Y_{s} d W_{s}^{(\mu)}\right)\right]
\end{aligned}
$$




$$
\begin{aligned}
& =\mathbf{E}_{0, y}\left[\exp \left(i t \int_{0}^{\tau_{a}} Y_{s} d W_{s}^{(\mu)}\right)\right] \mathbf{E}_{0, a}\left[\exp \left(i t \int_{0}^{+\infty} Y_{s} d W_{s}^{(\mu)}\right)\right] \\
& =\mathbf{E}\left[\exp \left(i t \int_{0}^{\tau_{a}} y e^{B_{s}^{(\nu)}} d W_{s}^{(\mu)}\right)\right] \mathbf{E}\left[\exp \left(i t \int_{0}^{+\infty} a e^{B_{s}^{(\nu)}} d W_{s}^{(\mu)}\right)\right] \\
& =\mathbf{E}\left[\exp \left(i t \int_{0}^{\tau_{a}} y e^{B_{s}^{(\nu)}} d W_{s}^{(\mu)}\right)\right] K(a t) .
\end{aligned}
$$

Thus, if we denote by $K_{a, y}$ the characteristic function of the hitting distribution on $H_{a}$, starting at $i y$, then

$$
K_{a, y}(t)=\frac{K(y t)}{K(a t)} .
$$

We already know that there exist sequences $\left\{a_{n}\right\}_{n},\left\{b_{n}\right\}_{n}$ of real numbers, with $a_{n}>0$, such that

$$
e^{-i b_{n} t} K\left(\frac{t}{a_{n}}\right)^{n} \underset{n \rightarrow \infty}{\longrightarrow} \phi(t),
$$

where $\phi$ is the characteristic function of a stable law, as described at the beginning of Section 5. Thus we have

$$
e^{-i(y-a) b_{n} t} K_{a, y}\left(\frac{t}{a_{n}}\right)^{n}=\frac{e^{-i b_{n} y t} K\left(\frac{y t}{a_{n}}\right)^{n}}{e^{-i b_{n} a t} K\left(\frac{a t}{a_{n}}\right)^{n}} \longrightarrow \frac{\phi(y t)}{\phi(a t)} .
$$

It is easy to check now that, if $\phi$ is the characteristic function of a stable law $S(z, c, \alpha, \gamma)$, then $t \longrightarrow \phi(y t) / \phi(a t)$ is the characteristic function of a stable law

$$
\begin{cases}S\left(z(y-a), c\left(y^{\alpha}-a^{\alpha}\right), \alpha, \gamma\right), & \text { if } \alpha \neq 1 \\ S(z(y-a)+c \gamma(y \log |y|-a \log |a|), c(y-a), 1, \gamma), & \text { if } \alpha=1 .\end{cases}
$$

Thus the law of $X_{\tau_{a}}$ is still in the domain of attraction of a stable law with exponent $\alpha=2 \nu$. More precisely, if $\nu<1$, if $\left\{X_{n}\right\}_{n}$ is a sequence of independent, identically distributed random variables with the same law as $X_{\tau_{a}}$, and if $m_{n}$ is defined as in Theorem 5.3, then

$$
\frac{X_{1}+\cdots+X_{n}-n m_{n}}{n^{1 / \alpha}} \stackrel{\text { law }}{\longrightarrow} m, \quad \text { as } n \longrightarrow \infty
$$


where $m$ is a stable law $S(0, c, \alpha, \gamma)$ with

$$
\begin{gathered}
\alpha=\min \{2,2 \nu\}, \\
c=2 c_{\mu, \nu}\left(y^{2 \nu}-a^{2 \nu}\right) C(2 \nu) \cosh \mu \pi, \\
\gamma=\tanh (-\mu \pi) .
\end{gathered}
$$

We omit the, otherwise obvious, statement for $\nu=1$.

\section{References.}

[AG] Alili, L., Gruet, J.-C., An explanation of a generalized Bougerol's identity in terms of hyperbolic Brownian motion. Exponential functionals and principal values related to Brownian motion. Ed. M. Yor. Biblioteca de la Revista Mat. Iberoamericana (1997), 15-33.

[BCF] Baldi, P., Casadio Tarabusi, E., Figà-Talamanca, A., Stable laws arising from hitting distributions of processes on homogeneous trees and the hyperbolic half-plane. Pacific J. Math. 197 (2001), 257-273.

[BLY] Biane, Ph., Le Gall, J.-F., Yor, M., Un processus qui ressemble au pont brownien. Séminaire de Probabilités XXI. Eds J. Azéma, P.-A. Meyer, M. Yor. Lecture Notes in Math. 1247 (1987), 270-275.

[CPY] Carmona, Ph., Petit, F., Yor, M., On the distribution and asymptotic results for exponential functionals of Lévy processes. Exponential functionals and principal values related to Brownian motion. Ed M. Yor. Biblioteca de la Revista Mat. Iberoamericana (1997), 73-130.

[EMOT] Erdélyi, A., Magnus, W., Oberhettinger, F., Tricomi, F. G., Higher transcendental functions Vol. I. Reprint of the 1953 original. Krieger, 1981.

[GR] Gradshteyn, I. S., Ryzhik, I. M., Table of integrals, series, and products. Academic Press, 1994.

[GK] Gnedenko, B. V., Kolmogorov, A. N., Limit distributions for sums of independent random variables. Addison-Wesley Math. Ser., 1968.

[H] Hoffmann-Jørgensen, J., Probability with a view toward statistics, I. Chapman \& Hall Probability Series, 1994.

[N] Nagahara, Y., The PDF and CF of Pearson type IV distributions and the ML estimation of the parameters. Statist. Probab. Lett. 43 (1999), 251-264.

[P] Paulsen, J., Risk theory in a stochastic economic environment. Stochastic Process. Appl. 46 (1993), 327-361. 
[PY1] Pitman, J. W., Yor, M., Bessel processes and infinitely divisible laws. Stochastic integrals (Durham 1980). Ed D. Williams. Lecture Notes in Math. 851 Springer (1981), 285-370.

[PY2] Pitman, J. W., Yor, M., Quelques identités en loi pour les processus de Bessel. Hommage à P. A. Meyer et J. Neveu. Astérisque 236 (1996), 249-276.

[RY] Revuz, D., Yor, M., Continuous martingales and Brownian motion. Grundlehren Math. Wiss. 293 Springer, 1999.

[T] Tricomi, F. G., Funzioni ipergeometriche confluenti. Monogr. Mat. 1 Cremonese, 1954.

[Y] Yor, M., Random Brownian scaling and some absolute continuity relationships. Seminar on Stochastic Analysis, Random Fields and Applications. Ascona, 1993. Eds E. Bolthausen, M. Dozzi, F. Russo. Progr. Probab. 36 Birkhäuser, 1995, 243-252.

Recibido: 29 de febrero de 2.000

Paolo Baldi

Dipartimento di Matematica Università di Roma Tor Vergata Via della Ricerca Scientifica 00133 Roma, ITALY baldi@mat . uniroma2. it

Enrico Cassadio Tarabusi, Alessandro Figà-Talamanca Dipartimento di Matematica "G. Castelnuovo" Università di Roma "La Sapienza" Piazzale A. Moro 2 00185 Roma, ITALY casadio@science.unitn.it sandroft@mat .uniroma1.it

Marc Yor Laboratoire de Probabilités, Université P. et M. Curie Tour 56, 4 Place Jussieu 75252 Paris, FRANCE deaproba@proba.jussieu.fr 1999". 